\title{
PENSAR LA (TEORÍA DE LA) EDUCACIÓN, DESDE LA TECNOLOGÍA DE NUESTRO TIEMPO' ${ }^{1}$
}

\author{
Thinking of (the Theory of) Education from the Technology \\ of our Time
}

Ángel GARCÍA DEL DUJO*, Joris VLIEGHE**, José Manuel MUÑOZ-RODRÍGUEZ* y Judith MARTÍN-LUCAS*

*Universidad de Salamanca. España.

agd@usal.es; pepema@usal.es; judithmartin@usal.es

bttps://orcid.org/0000-0002-5202-7366; https://orcid.org/0000-0002-4688-6420;

https://orcid.org/0000-0002-1655-0280

*Katholieke Universiteit Leuven. Bélgica.

joris.vlieghe@kuleuven.be

https://orcid.org/0000-0001-6307-3221

Fecha de recepción: 11/01/2021

Fecha de aceptación: 21/02/2021

Fecha de publicación en línea: 01/07/2021

Cómo citar este artículo: García del Dujo, Á., Vlieghe, J., Muñoz-Rodríguez, J. M., y Martín-Lucas, J. (2021). Pensar la (teoría de la) educación, desde la tecnología de nuestro tiempo. Teoría de la Educación. Revista Interuniversitaria, 33(2), 5-26. https:// doi.org/10.14201/teri.25432

1. Esta investigación se inscribe dentro de los siguientes proyectos financiados: 1) «CONECT-ID. "La identidad hiperconectada de la juventud y su percepción del tiempo en el ocio digital". Ministerio de Ciencia, Innovación y Universidades. Referencia: PGC2018-097884-B-I00 (2019-2022). IP. José Manuel Muñoz-Rodríguez. 2) "Identidades digitales en jóvenes hiperconectados: retos para el contexto familiar, social y escolar. Junta de Castilla y León. Referencia: SA038G19 (2018-2021). IP. José Manuel Muñoz-Rodríguez. 


\section{RESUMEN}

En (teoría de la) educación necesitamos abordar cuanto antes una pregunta: ¿Cómo hacer y pensar la educación en consonancia con las características, exigencias y oportunidades, de esta época digital? A este respecto, después de referenciar los derroteros que en este campo viene siguiendo la investigación pedagógica y la práctica educativa, se plantean en el cuerpo del artículo tres cuestiones - modos de ser, de experienciar el mundo y de construir conocimiento en un sujeto cuya relación con el entorno es mediada por una tecnología cuyo rasgo definitorio viene dado por una pantalla digital — que están demandando otra forma de ver la educación, dicho de otra manera, tres cuestiones directamente afectadas por el nuevo escenario creado por estas tecnologías y que una (teoría de la) educación de nuestro tiempo no puede ignorar, pues sería tanto como no (querer) ver la transición que se está produciendo en las formas de pensar y hacer educación; de alguna manera, sobre ellas descansa y pivota una (teoría de la) educación acorde con este nuestro tiempo digital.

El artículo termina con un elenco de preguntas de orden diverso, epistemológico, ontológico y metodológico, pero también ético y politico, estrechamente entrelazadas, que dejan entrever la conveniencia y necesidad de reorientar la reflexión en este campo, al tiempo que conforman un programa de investigación; preguntas con potencialidad suficiente para reorientar la investigación en (teoría de la) educación, inluido el propio concepto y sentido de educar y ser educado.

Palabras clave: pantallas digitales; reontologización de la realidad; percepción y construcción del yo; construcción digital de conocimiento; entornos y gramáticas digitales.

\section{ABSTRACT}

In (theory of) education we must address one question as soon as possible: how to create and think of education in keeping with the characteristics, demands and opportunities of this digital age. In this regard, after referring to the paths pedagogical research and educational practice have been following in this field, three questions are raised in the body of the article-ways of being, of experiencing the world and of creating knowledge of a subject whose relationship with the environment is mediated by a technology whose defining feature comes from a digital screen-which are demanding a new way of looking at education. In other words, three issues directly affected by the new scenario created by these technologies and which a (theory of) education of our times cannot ignore as that would amount to not (wanting) to see the transition occurring in ways of thinking and putting education into practice; the (theory of) education in our digital time somehow rests on or revolves around them.

The article ends with a range of diverse, closely intertwined questions: epistemological, ontological, methodological, but also ethical and political, which give us a glimpse of the advisability or need to redirect thought in this field while forming a research programme; questions with sufficient potential to redirect (theory of) education research, including the very concept and meaning of educating and being educated.

Key words: digital screens; reontologisation of reality; self-perception and construction; building digital knowledge; digital environments and grammar. 
ÁNGEL GARCÍA DEL DUJO, JORIS VLIEGHE, JOSÉ MANUEL MUÑOZ Y JUDITH MARTÍN

PENSAR LA (TEORÍA DE LA) EDUCACIÓN, DESDE LA TECNOLOGÍA DE NUESTRO TIEMPO

\section{INTRODUCCIÓN}

Hace ya un tiempo decíamos que estas tecnologías, que muy pronto dieron en llamarse de la información y la comunicación porque básicamente así lo eran en su primera infancia, al tiempo que proporcionaban a la educación un buen número de posibilidades y oportunidades, planteaban también a la pedagogía algunos requisitos y exigencias; entre otros, nos referíamos a la conveniencia, y hasta necesidad, de pensar la educación, los agentes, espacios y procesos de formación, de otro modo (García del Dujo y Martín-García, 2002; 2005).

No han sido éstos, sin embargo, los derroteros que en este campo ha seguido la investigación pedagógica y la práctica educativa; al contrario, en ambos casos, tanto en la investigación como en la práctica, así como en los programas de innovación educativa, ha predominado un mismo movimiento, dirección y concepción de esta tecnología, implementado en su doble sentido: de un lado, se ha producido una transposición lineal de las prácticas tradicionales a los nuevos espacios que generan estas tecnologías, aceptando por principio, y sin mayor cuestionamiento, que estas tecnologías reproducen los espacios, agentes y procesos de pensamiento tradicionales, y, de otro, se ha buscado la integración de estas tecnologías en los espacios tradicionales como un instrumento más al servicio de esos espacios, agentes y procesos formativos tradicionales, sin caer en la cuenta de que introducir un nuevo componente en un ecosistema termina cambiando el comportamiento del sistema, sobre todo si ese nuevo elemento viene dado por una tecnología diferente. El orden con el que se ha producido en el tiempo este doble movimiento fue, primero, la consideración instrumental de estas tecnologías al servicio de los enfoques vigentes de enseñanza y, en un segundo momento, la traslación literal de la perspectiva tradicional del proceso de enseñanza-aprendizaje al espacio virtual.

Ambos momentos e interpretaciones confluyen en una concepción instrumental, escolar y didactista, instruccional, individualista, de estas tecnologías, una concepción reduccionista de su enorme potencial educativo a simples herramientas de producción, almacenamiento, traslado y gestión de información y comunicación, cuyo culmen y mejor reflejo en el ámbito educativo probablemente se haya dado en tiempos de confinamiento obligado por esta pandemia; una concepción manifiestamente insuficiente y mejorable, como lo demuestra el grado de insatisfacción que en estos tiempos convulsos han generado en profesores y estudiantes los procesos de formación así planteados, sin perjuicio de reconocer que peor hubiera sido el escenario en ausencia de esta tecnología. ¡Por supuesto!

Este planteamiento que venimos haciendo se aprecia a primera vista en cualquiera de las numerosas revistas científicas que publican trabajos que giran en torno a esta concepción como medios, muchas de las cuales nacieron al tiempo y al amparo de estas tecnologías y han evolucionado temáticamente al ritmo generacional que ellas vienen marcando, de manera que contamos hoy con una amplísima literatura a propósito de una tecnología que progresivamente va dejando 
ÁNGEL GARCÍA DEL DUJO, JORIS VLIEGHE, JOSÉ MANUEL MUÑOZ Y JUDITH MARTÍN PENSAR LA (TEORÍA DE LA) EDUCACIÓN, DESDE LA TECNOLOGÍA DE NUESTRO TIEMPO

ver su potencialidad educativa, si bien la pedagogía la reinterpreta, una y otra vez, siempre en la misma dirección, ya sea como medios susceptibles de análisis comparado con otros medios, como medios que despiertan determinadas actitudes en los usuarios, ya sean docentes o discentes, como medios que requieren ciertas competencias, como medios de formato uniforme con validez multidisciplinar o diverso en función de las características de cada tecnología más que en función de las exigencias pedagógicas... (Cabero, 2016); todo lo más se han ido abriendo y acogiendo en el diseño instruccional principios de colaboración, cooperación,... y otros aspectos metodológicos en consonancia con las versiones actualizadas del principio vigotskiano de la actividad, mediada ahora por esta tecnología, y de los principios socioconstructivistas reformulados en sucesivas generaciones.

Bien podríamos pensar, en consecuencia, que esta forma de interpretar y usar la tecnología nos sigue llevando a pensar y hacer educación desde una concepción moderna (tradicional) de la enseñanza y del aprendizaje, una concepción escolar de la educación, por más que entren en juego herramientas propias de una sociedad postmoderna. El esquema mental de lo educativo ahí implícito sigue respondiendo a una manera de entender la realidad propia de la modernidad, como si esta tecnología no hubiera cambiado las formas de ver, de hacer y de pensar esa realidad, incluidas, por supuesto, las formas de pensar y hacer educación.

Hay, en cambio, otra posible interpretación de esta tecnología que interesa más a la teoría de la educación; esta otra lectura toma como punto de apoyo la consideración de la tecnología como cultura, en el sentido de que crea formas de pensar, de ser y de actuar, en definitiva, formas de vivir y mundos de vida diferentes, en cuanto que cambia las formas de relación con las cosas y con los otros, terminando por cambiar también los modos de percepción y creación de realidades, incluida nuestra propia identidad y la de las cosas del mundo; es en este doble sentido - desde las cosas y desde la tecnología- que varios autores hablan de un proceso de reontologización de la realidad catalizado por las tecnologías de nuestro tiempo (Floridi, 2014 y Vlieghe, 2019, si bien lo hacen en diferente sentido). Y, en consonancia con este otro planteamiento, cabe pensar - más aún, conviene pensar- que también la educación puede y debe quedar afectada, pero no solo en el sentido de que pueda ser conseguida de otra manera, por otros medios, sino en el sentido de su misma interpretación y concepción.

Pues bien, en este artículo nos proponemos someter a reflexión tres cuestiones que estarían demandando esa otra forma de ver la educación, dicho de otra manera, tres cuestiones directamente afectadas por el nuevo escenario creado por estas tecnologías y que una (teoría de la) educación de nuestro tiempo no puede ignorar, pues sería tanto como no (querer) ver la transición que se está produciendo en las formas de pensar y hacer educación, incluido el propio concepto de educación; cuestiones que hacen referencia a componentes educativos - modos de percepción, espacios, prácticas y relaciones, agentes y procesos de construcción del 
ÁNGEL GARCÍA DEL DUJO, JORIS VLIEGHE, JOSÉ MANUEL MUÑOZ Y JUDITH MARTÍN

PENSAR LA (TEORÍA DE LA) EDUCACIÓN, DESDE LA TECNOLOGÍA DE NUESTRO TIEMPO

conocimiento- que, aportados y catalizados por esta tecnología, están pidiendo una resignificación global de la educación. Y terminaremos con un elenco de interrogantes que concretan esta otra forma de ver la (teoría de la) educación, si bien lo hacen en forma de preguntas.

\section{EXPERIENCIAR EL MUNDO A TRAVÉS DE UNA PANTALLA - LA EXPERIENCIA DE MIRAR EL MUNDO A TRAVÉS DE UNA PANTALLA...}

En esta sección, se extraen las implicaciones ontológicas del cambio hacia la educación digital. Se argumentará que, cuando dependemos cada vez más de dispositivos digitales, y, más exactamente, cuando el mundo se manifiesta a través de las pantallas, existe algo nuevo y único en relación con la realidad. Lo que tiene, a su vez, graves consecuencias para la forma en que educamos y la forma en que deberíamos teorizar la educación en el siglo XXI.

No hace falta decir que el fenómeno de la digitalización en la educación (y en otras esferas de la vida) puede analizarse desde muchos ángulos diferentes. Un ejemplo de ello es el cambio drástico de lo que significa relacionarse con el conocimiento, donde se puede afirmar que nos hemos liberado finalmente de la acumulación inútil de detalles y hechos, ya que la información está disponible siempre y en cualquier lugar, pero también que ya no procesamos el conocimiento a un nivel lo suficientemente profundo como para realizar cambios significativos en nuestras vidas. Otro ejemplo sería llamar la atención sobre las nuevas posibilidades y limitaciones relativas a los aspectos sociales de la vida. Con el uso masivo de las herramientas digitales se ha hecho físicamente posible formar parte de colectivos que se expanden más allá de cualquier dimensión social que la humanidad ha conocido hasta ahora (según lo que Robin Dunbar -2010 — afirma que es la capacidad máxima del número de personas con las que podemos conectar para formar un verdadero 'grupo' - una cifra en torno a 150, basada en su estudio de las formas de vida de los primates, el tamaño de la ciudad neolítica, las unidades básicas del antiguo ejército romano, etc.). Pero, de nuevo, el mismo hecho puede ser abordado críticamente, argumentando que todo esto conlleva nuevas formas de tribalismo, donde cada uno está constantemente expuesto a la mirada del otro y se espera continuamente que comparta contenido y responda a lo que los demás publican en línea, lo que trae consigo el fin de la mentalidad moderna (que es antitribalismo puro y duro, ya que está dirigido a aislar y emancipar al individuo del grupo, con la ayuda de medios políticos, urbanísticos y, sobre todo, educativos; véase Fogel y Patino, 2013).

A continuación, abordaremos la digitalización desde un punto de vista específico y triple. En primer lugar, y contrariamente a los dos ejemplos que acabamos de ofrecer, el objetivo no es desarrollar una posición normativa (es decir, si debiéramos o no acoger la digitalización). La digitalización se considerará simplemente como la condición en la que vivimos y educamos hoy día. Esto implica que las consecuencias 
de tal cambio en las condiciones deben ser cuidadosamente extraídas. Pero, conforme a los dos ejemplos que acaban de exponerse, en este artículo se da por sentado que la introducción de los nuevos medios de comunicación tiene efectos de gran alcance en la forma en que se estructura la realidad (humana): viene acompañada de profundos cambios ontológicos (por ejemplo, trasmutando la esencia misma de lo que puede ser un colectivo verdadero).

En segundo lugar, se da un paso más en este punto de vista, ya que se afirmará que la digitalización equivale a reorganizar nuestra comprensión de la realidad como tal. El cambio que se discute en esta sección va más allá de una simple alteración de las estructuras ontológicamente relevantes: lo que está en juego es que en un mundo digital lo que cuenta como real sufre un cambio drástico. Para ser más precisos, en condiciones digitales la realidad se experimenta como totalmente inmanente (Vlieghe, 2019). Para fundamentar tal afirmación, es importante, en tercer lugar, abordar lo digital desde un ángulo específico, a saber, que el mundo se nos muestra cada vez más a través de una pantalla. Vivimos en un mundo en el que las pantallas se han hecho omnipresentes, hasta tal punto de que, para las nuevas generaciones, se ha vuelto casi impensable mostrar el mundo en el que viven sin la mediación de la pantalla (Tisseron, 2010). Por lo tanto, es de suma importancia entender cómo la realidad emerge en la pantalla plana y rectangular de nuestros aparatos digitales, que son cada vez más portátiles.

Cuando abordamos esta cuestión desde una comprensión modernista de las tecnologías estéticas (como los dibujos y las pinturas), es decir, cuando definimos la pantalla como una ventana que da acceso al mundo que hay detrás de ella, podemos estar profundamente equivocados — aunque esta imaginería ha sido más influyente desde el Renacimiento y especialmente a raíz de la discusión de Alberti sobre ella en De Pictura (1435). Al menos, esto es lo que argumenta Mauro Carbone, que define la ventana y la pantalla como dos dispositivos metafísicamente opuestos (Carbone 2013). La ventana, de hecho, nos transporta desde la esfera del aqui a otro reino del allá. La pantalla, por el contrario, cuando se apaga, se convierte en un espejo (no accidentalmente), nos devuelve a nosotros mismos. Esto es aún más cierto cuando miramos la pantalla de nuestros teléfonos inteligentes: no estamos mirando a través de nuestra mano a una esfera más allá de ella. En su lugar, tenemos una experiencia de nuestros sentidos visuales y táctiles fusionados en la misma palma de nuestra mano. Lo que vemos coincide con lo que sentimos capturado en nuestra mano. Por eso Heidi Cooley (2004) identifica correctamente esta experiencia en términos de visión táctil.

Pues bien, todo esto viene con un efecto de realidad particular. O lo que es lo mismo: lo que vemos, y, por lo tanto, también sentimos aparecer en la pantalla cuenta igual de real (o incluso más real) que el mundo al que se accede de otra manera. Esto explica la ausencia de dificultad de muchos para mirar en una pantalla cuando la hay, o por qué podemos visitar un museo con el único objetivo de 
capturar imágenes de los objetos de interés que allí se encuentran. Mientras que en tiempos predigitales la fotografía era un instrumento para representar objetos en su ausencia física, donde el medio (la cámara) no era más que un medio para crear una imagen que existe con independencia de este medio (fotografías impresas), hoy en día podemos hacer (producir) y ver (consumir) imágenes con un mismo dispositivo, con el objetivo principal de tenerlas capturadas en nuestro dispositivo portátil. Queremos que estén presentes a mano en el sentido más absoluto de esa palabra (mientras que las imágenes impresas se han convertido en algo sin sentido). En francés se dice: queremos que la realidad sea main-tenant (que significa tanto "ahora" como "contenido-en-mi-mano"; Serres, 2012). Únicamente nos interesa lo que podemos experimentar aquí y ahora, en la palma de nuestra mano. Y, lo que es más importante, esto significa que, en lugar de anhelar una realidad fuera o en el exterior (como era el caso en el moderno aparato de la ventana), queremos relacionarnos con el mundo de una manera totalmente inmanente. La única realidad que cuenta es el aquí y el ahora radical —el Maintenant. Se podría argumentar que el mismo análisis se aplica a todas las pantallas, las portátiles y las fijas, las digitales y las no digitales. Las pantallas de nuestros dispositivos digitales deben considerarse entonces como la expresión más perfecta de la esencia de screeness que únicamente se alcanzó parcialmente en los formatos de pantalla más antiguos (véase Introna \& Ilharco, 2006; Vlieghe, 2019).

Ahora, nuestra nueva condición ontológica de Maintenant, resultante de relacionarnos con el mundo en la pantalla, puede ser dilucidada aún más volviendo a sus orígenes premodernos. Tenemos que remontarnos a la antigüedad romana para encontrar el origen del marco que hoy llamamos pantalla: yendo en contra del relato más popular de que la pantalla funciona de la manera en que lo hace la ventana y cuestionando que esto explicaría el hecho de que las pantallas, con algunas excepciones, son rectangulares, Anna Caterina Dalmasso (2020) sostiene que las pantallas se originan dentro de la práctica del augurio, donde el sacerdote, llamado a predecir el futuro, tenía que realizar el gesto templun, es decir, usar sus pulgares e índices para formar cuatro ángulos rectos que circunscribían un campo rectangular dentro del cual se debía observar y estudiar el vuelo de los pájaros. Se tenía que circunscribir una porción de los cielos para poder leer los signos que podían predecir el destino de los romanos.

Es importante señalar que esta forma de predecir el futuro puede considerarse un gran paso adelante respecto a las antiguas prácticas adivinatorias: mientras que los romanos solían basarse en la siempre subjetiva y arriesgada interpretación de los signos no concluyentes, y especialmente en las predicciones de oráculos equívocos y arcanos, con el advenimiento del augurio la adivinación se hizo más objetiva. Esto se debe a que existe una brecha insalvable entre lo que el sacerdote del oráculo predice y los eventos que ocurrirán en el futuro. Por el contrario, se creía que había una conexión directa e inmanente entre los vuelos de los pájaros y los eventos 
futuros, porque ambos estaban causalmente ajustados al orden causal de la naturaleza. En ese sentido, el augurio era más objetivo y un primer paso hacia lo que hoy llamaríamos una visión científica del mundo. Pero, lo que es más importante en el contexto de este artículo, Dalmasso sugiere que el templum es un dispositivo que permite una forma particular de ver el mundo que es totalmente inmanente (y que fue olvidado o reprimido, sugiere Dalmasso, porque desde la Modernidad hemos empezado a pensar en términos de la ventana como el dispositivo dominante para representar el mundo).

Además, vivir en las condiciones de un Maintenant radical también conlleva una reestructuración de nuestra experiencia sobre lo que significa estar situado en el tiempo, es decir, de qué se trata el tiempo. Una vez más, es útil oponerse a una forma modernista de ver lo que ocurre cuando la pantalla digital se ha vuelto omnipresente. En línea con la metáfora de la ventana moderna, ligada a la actividad de mirar a través, es decir, hacia adelante, podría decirse que la experiencia temporal a la que nos hemos acostumbrado es una experiencia de futuro. Con el trasfondo de una ideología moderna de progreso, estábamos acostumbrados a situarnos en una línea del tiempo que se extendía desde un pasado ya pasado hasta un futuro todavía no presente (y con suerte más brillante). Por lo tanto, el pasado es literalmente lo que está detrás de nosotros, mientras que el futuro es la dimensión que está delante. El futuro es lo que tenemos delante. Pero, cuando el mundo se revela a través de la pantalla, lo que tenemos delante ya no es el futuro. El contenido que aparece en la pantalla es presente (como en la transmisión en directo) o pasado, como es mayormente el caso (es decir, contenidos desarrollados y diseñados antes de que nuestros ojos los vean). El usuario de la pantalla ha dejado, por así decirlo, cualquier orientación hacia el futuro y, en ese sentido, el futuro está detrás de él o de ella; en cambio, lo que está delante de él o de ella es el presente y lo que ya ha sido presente —el pasado (Véase Fogel \& Patino, 2013). Como resultado, acabamos con una experiencia ontológica del tiempo completamente diferente que, de acuerdo con nuestro análisis hasta ahora, debería ser llamada totalmente inmanente. No se requiere ninguna relación con una dimensión fuera o más allá del aquí y ahora para percibir las cosas como relevantes y significativas, y sobre todo como reales.

Haciendo referencia a las reflexiones de Vilém Flusser (2011) sobre los medios digitales, esta nueva forma de experimentar el tiempo también podría llamarse poshistórica. Flusser sostiene que era de suma importancia para quienes vivían bajo condiciones modernas, es decir, predigitales, situarse en la historia y encontrar un terreno firme en ella: necesitábamos saber dónde estamos en el tiempo, en qué época vivimos, qué épocas hemos dejado atrás y qué podemos esperar razonablemente que suceda en el futuro. Ahora, en un mundo totalmente digitalizado, tal visión ha perdido todo su significado. Situarnos en una posición dentro de una trayectoria histórica ya no nos permite dar dirección a nuestra relación con el mundo. Lo que cuenta es, de nuevo, exclusivamente el aquí y el ahora. 
Curiosamente, para Flusser la forma histórica de relacionarse con el mundo está conectada con otra gran metáfora de la modernidad (junto a la ventana): el libro. Leemos los libros de principio a fin, dedicando largos períodos de concentración sostenida, y los leemos en un orden progresivo — sintiendo manualmente cómo progresamos a través de ellos como resultado de la presión desigualmente repartida que se percibe en nuestras manos izquierda y derecha. La práctica de la lectura apoya así una experiencia temporal orientada al futuro y al progreso. Ahora bien, el libro también podría llamarse el Leitmedium de la educación moderna, como sugiere Jeanette Böhme (2006). El comienzo de la Modernidad también coincide con la invención y la rápida proliferación de textos impresos, forzando a la sociedad a un programa a gran escala de instrucción de los jóvenes en la lectura y la escritura, que ahora llamamos educación escolar. En la época del libro, ser analfabeto es tan oneroso como carecer de vista y oído - la cosa está en que una educación que está a la altura de su época se ocupa de iniciar a la nueva generación en el medio culturalmente dominante para acceder al mundo, es decir, el Leitmedium (cf. Stiegler, 2008). Asimismo, en una época en la que tenemos que ocuparnos de la educación de los screenagers, sería conveniente tener en cuenta que nos enfrentamos a una nueva tecnología dominante de divulgación del mundo, e incluso a una que enmarca la propia realidad de formas completamente diferentes a las que solía.

Esto implica más concretamente dos cosas. Primero, que debemos asumir que los conceptos educativos básicos han cambiado de una manera fundamental y que no deben ser juzgados en función de estándares predigitales pasados. A modo de ejemplo: la noción de creatividad adquiere un significado completamente diferente en vista de lo que se ha dicho anteriormente. Cuando pedimos a los estudiantes que escriban un ensayo, tal vez busquemos, por ejemplo, un trabajo que no sea un mero y 'clásico' informe de investigación escrito, sino que consista en seleccionar, repasar, enredar y reelaborar materiales digitales existentes. Y podríamos querer validarlo como original, lo que significa que tenemos que redefinir otra noción ontológica importante, a saber, la novedad. En segundo lugar, si nos enfrentamos a un nuevo Leitmedium educativo, esto también implica que queremos adquirir competencia en el uso de la nueva tecnología digital, así como ser capaces de relacionarnos con ella de forma crítica. Esto implicaría un cambio en el plan de estudios centrado en lo que podría llamarse una ortografía y gramática de lo digital.

\section{TÚ, YO Y LAS COSAS, EN LOS ESPACIOS VIRTUALES - ... DE VERNOS A NOSOTROS MisMos MiRANDO AL MUNDO A TRAVÉS DE UNA PANTALLA...}

También tú y yo, en cuanto realidad que somos, estamos sometidos a procesos de transfiguración cuando miramos el mundo y habitamos en estas pantallas. Recordaremos a este respecto, de un lado, que toda tecnología, en el sentido de cultura que aquí interesa, lleva consigo una cierta re-significación de las maneras como soy y como pienso, las formas de relacionarme con los demás, con el propio 
entorno y conmigo mismo, pues se reconfiguran los mecanismos a través de los que veo las cosas que me rodean, hago y cambio esas cosas, las uso o simplemente las desecho, quedando implicados procesos de adaptación al nuevo entorno cultural, de construcción y reconstrucción de la propia identidad, individual y social, y procesos de intersubjetividad en los que los humanos nos hacemos. Nuevos sucesos culturales que suscitan interrogantes de interés educativo: ¿de qué manera las formas de pensar sobre mí y sobre los demás, de mirar y de hacer cosas con ellos quedan afectadas, alteradas, recompuestas por esta tecnología nuestra?, ¿hasta qué punto los procesos de autonomía, autorregulación y responsabilidad, emoción y afecto, pensamiento crítico, relación y socialización, requieren una relectura a la luz de la tecnología predominante en esta época?

Por otra parte, leer en perspectiva educativa la tecnología de hoy, los entornos virtuales que genera, aconseja observar e interpretar lo que ahí pasa en clave humana, los quehaceres vitales que ahí acontecen. Leer en el rostro del yo, de los otros, de las cosas que hay entre ellos y del entorno que lo envuelve todo, para descubrir trazos de humanización, de necesidad vital, como base de lo educativo. No se requiere gran experiencia técnica para advertir que, en las redes sociales, en los juegos online, en las plataformas virtuales, hay vida, afectos y emociones, y cosas, artefactos culturales que propician el desarrollo humano y, sobre todo, gentes que activan y experiencian procesos de desarrollo. Conviene, por tanto, hacer esa relectura desde el sentido que plantea la pregunta por la condición humana en lo tecnológico, identificando los límites y posibilidades de ese desarrollo en, con y desde esa tecnología, pues la pretensión implícita es descubrir ahí la necesidad vital como catalizadora de los procesos educativos que configuran el ser humano, por complejo y extraño que parezca al observar en numerosas ocasiones, más que procesos de humanización, procesos de deshumanización con origen en relaciones efímeras, discontinuas, pasajeras.

Pues bien, de acuerdo con estas premisas, podríamos enunciar el punto de partida en este epígrafe en los siguientes términos: la naturaleza humana — siendo ella también cultura- y la cultura confluyen, se necesitan y reconfiguran cuando los escenarios de vida están mediados tecnológicamente, sea cual fuere la tecnología de referencia, y, por tanto, desde una concepción de cultura como proceso primario y desde esa indisociable realidad de la especie humana, naturaleza y cultura a la vez, algo de esto tiene que ocurrir también en los escenarios virtuales. En este sentido, la investigación viene demostrando que, más allá del artefacto tecnológico concreto, en la web se entrelazan elementos y se conforman experiencias que se visualizan mediante patrones de actividad, acción y relación (Pangrazio, 2019). Se generan sitios, que se transforman en lugares, se intercambian cosas, se expresan sentimientos, se transmiten valores, aunque siempre sea en base a información; hay vida, con comunidades sociales y cosas en los alrededores de esas comunidades que catalizan, dinamizan y hacen visibles procesos de construcción de identidades, individuales y colectivas. De manera que, al igual que se da una re-ontologización de la realidad mirando a través de las pantallas, que conlleva una importante 
re-significación, incluyendo dimensiones espaciales y temporales, también se dan procesos de construcción y reconstrucción de identidades que procede entender y atender desde la educación. Vayamos, pues, por partes.

Observemos el yo y los procesos intrasubjetivos. El sujeto se mira a sí mismo, se escucha, se percibe en sus propias imágenes, se hace oír hablándose a sí mismo a través de los múltiples perfiles que puede mostrar en la red, conformando todo ello el procesamiento de la experiencia personal expuesta en términos de gramáticas particulares, representadas a través de los lugares que escoge, imágenes que comparte, los likes que muestra o los sitios que evita, y de las gramáticas propias de lo digital que condicionan la conformación del yo y del tú. Es la narratividad de la publicidad-privacidad-intimidad; en función de dónde y cuándo se sitúa, el sujeto gestiona su yo, su publicidad y/o privacidad de una u otra manera, utilizando uno u otro perfil en la búsqueda de la construcción del yo, de la privacidad e incluso de la intimidad que desea (Schofield \& Kupiainen, 2015). El problema viene cuando en unos sitios el yo se comporta y presenta un perfil distinto al que muestra en otros lugares (Greenfield, 2014). En la vida offline la preocupación era qué aspectos de nuestra vida decidíamos que fueran íntimos; ahora, en la vida online, el reto es qué hacer para lograrlo una vez que lo hemos decidido, pues la intimidad se reconceptualiza, más aún, podemos llegar a mentir en nuestra identidad personal, cambiar de nombre, de edad, de raza, presentar un yo imaginario. La transversalidad y multiramificación de la red permite mostrar diferentes yoes que, a su vez, coadyuvan al individuo a satisfacer necesidades y deseos que en entornos presenciales nunca hubiera podido realizar de manera intencional. Así, gestionar la visibilidad de uno mismo es gestionar el proceso de construcción del yo, un proceso que permite mostrar diferentes identidades y conjugarlas según el sitio y el momento en que nos estemos mostrando. Son varios y complejos los procesos de identidad que se activan apoyándose en la imagen, que no siempre es la deseada, ni la real, con el riesgo incluso de dañarla, pues tampoco suele mostrarse desde la unidad, sino más bien desde la fragmentación, la discontinuidad, derivando en situaciones indeseadas (Memon et al., 2018), sobre todo cuando con frecuencia no responde a intención consciente, sino más bien compulsión incontrolada.

La tecnología como cultura es vida, la diversifica y la amplía, pero a veces también la complica, creando dependencias que derivan en inseguridades ontológicas adheridas a vidas frágiles, contingentes e inciertas, carentes de procesos reflexivos y de pensamiento crítico, aspectos que forman parte del argumentario sobre el que se fundamenta la no natividad digital de los jóvenes, todo lo cual está apuntando a la necesidad de poner en marcha procesos educativos orientados a la autoreflexión, no solo a la autoexpresión (Mace, 2020).

Miremos ahora el tú, los otros, los procesos intersubjetivos. El ser humano digital necesita del tú para comprobar que está y existe en la red, para reafirmar quién es realmente, para aceptarse como es y entender lo que le pasa, lo que hace y lo que siente. La red no solo le permite ser escuchado y sentirse acompañado, sino que modifica, desde la posición del grupo, las formas como se es y se está en 
un grupo, consecuencia también de la superposición de redes, y así va adquiriendo el sujeto autonomía y reflexividad, desde los comportamientos individuales en la red, aunque también es la colectividad quien marca el proceso de autorregulación, pues la tecnología, entendida como cultura, es básicamente social. No son solo instrumentos para ser usados, sino sitios conformados técnicamente para la creación de comunidades sociales, sitios que se vuelven lugares a través de acciones, sentimientos, juegos, generando así otros sitios y lugares; precisamente ahí radica la importancia y necesidad de no olvidar, en cualquier reflexión que hagamos en perspectiva educativa, los límites que vienen dados por las gramáticas digitales, la anotomía y fisiología de lo digita, y que terminan conformando identidades y comportamientos.

Observando la vida online, se perciben procesos de negociación de sentidos de identificación colectiva en los grupos, lo que supone salir del yo y reafirmar su inmanencia en una posición de igual a igual, colectiva, cuya representación social viene descrita por la búsqueda de acuerdos, relaciones y subordinaciones de distinto signo respecto de otros, como muestra de su capacidad de expresión social. Unas manifestaciones que se hacen visibles en forma de gestos, miradas, comentarios, fotos, paisajes y un largo etcétera de representaciones sociales que conforman y confirman el yo social en la red. Es así como el entorno se convierte en un territorio social simbólico, donde el grafismo y la imagen compartida constituyen los componentes centrales de los procesos de comunicación y relación que en él se activan y desarrollan a través de miradas y lecturas visuales hipertextuales, nunca lineales, y que dan lugar a procesos de desterritorialización y reterritorialización constantes. Es lo que Goffman (2001) denominó gestión de impresiones que el yo quiere captar del tú, construyendo en este caso una red de relaciones que supera lo que institucional y socialmente ha venido estando establecido, generando mecanismos de agregación de iguales en determinados entornos y a veces otros entornos, desde la negociación de una identidad colectica permanente. La idea de colectivo común online, que comparte ideas, atributos, aficiones, intereses, lo permite al adquirir un sentimiento de compartición de vida con el tú; se amplían, se estrechan, se diversifican los criterios de demarcación social, reconstruyéndose el yo con muchos otros en diferentes y heterogéneos ámbitos de la vida social.

Ahora bien, de nuevo aquí, al igual que en los procesos intrasubjetivos, existe riesgo de encontrarnos con superficialidad en la relación, suplantando los procesos básicos de socialización por otros que tienen más que ver con la ficción, asociada al aislamiento social más que a la sociabilidad. La investigación a este respecto habla incluso de saturación social a la que el uno se somete permanentemente, consecuencia de una amplificación relacional asociada proporcionalmente a la desconexión real del otro (Costa, 2018), detectando aquí un nicho de oportunidades para el desarrollo de una educación básica asociada a procesos adaptativos al nuevo medio y entorno tecnocultural.

Y, en último término, observemos el todo, el tú, el yo y las cosas: procesos de interdependencia vital asociados a acciones entre los grupos mediante cosas que se 
hacen, se utilizan, se construyen y consumen en esos grupos. La literatura al respecto muestra interés en resaltar el carácter del sujeto digital como sembrador y recolector, productor y consumidor de cosas — artefactos, juegos, anuncios, comentarios, imágenes, ideas, respuestas, relaciones, información, etc.- (Sánchez Chamorro et al., 2020), en definitiva, historias que terminan de construir su yo desde la unidad y la estabilidad. La relación, comunicación, afectos manifestados, adquieren pleno sentido en la in-mediatez cuando son mediadas por la tecnocultura en la que se insertan, por las cosas, asociadas a entornos, con las que conviven y dan unidad a la comunidad desde un diálogo tripartito.

Son las cosas las que terminan de dar apetencia al espacio y al tiempo, al lugar y momento escogidos para entrar y quedarme en la red. Aquello que permite al yo y al tú sentir, proyectar, actuar..., ser y estar, desde el diálogo con el momento y con las cosas del entorno. La lógica y valor de lo compartido no se genera y afecta solo al yo y al tú, no es solo lo social por lo social, sino que también entra en juego, con un rol muy importante, el diálogo con las cosas que se ven, se hacen, se crean, se consumen. Un diálogo que en la red es más bien puntual, adherido a pertenencia a lugares, momentos, paisajes tecnoculturales que vivimos y compartimos (Côté \& Levine, 2002); es la soledad entendida del revés, compartida, amplificada, aunque en la ventana estés tú solo asomado.

Si resulta necesario revivir los lugares que marcaron nuestra infancia, por poner un ejemplo, porque allí no solo hubo gentes sino también cosas que ciertamente se precisan volver a ver y disfrutar, no es menos cierto que los espacios virtuales también habilitan esa reminiscencia de las cosas, construidas o compartidas, que en un momento conformaron nuestra identidad. Lecturas, lugares, paisajes, deportes, juegos, aficiones o gustos, que hablan de vínculos humanos, no siempre puestos a la vista, ni fáciles de encontrar, pero que siempre están y existen. Es un nuevo concepto de espacio público, donde las cosas que hago y comparto me permiten hablar de cotidianeidad en mi vida y de rutinas en las nuevas calles y en los nuevos barrios (Humphreys et al., 2013).

Y de nuevo aquí encontramos límites y oportunidades para la educación, pues no siempre se cuidan y valoran las cosas, sino que se usan de manera efímera y se tiran, no se interiorizan, no se cultivan. Y el mundo de vida del sujeto pasa así a ser también un mundo efímero, discontinuo, líquido, un mundo de relaciones sin formas estables, transitorias, evanescentes, fácilmente mutables, lo que nos advierte, al igual que ocurría en los procesos anteriores, la pertinencia de reconceptualizar lo educativo para pensarlo desde la necesidad de acompasar los tiempos y los espacios, los mundos de vida on y offline; éste es el concepto de experiencia, vida e identidad onlife (Floridi, 2014), un cambio ontológico que debe empezar por desaprender lo que la tecnología viene imponiendo desde sus inicios: vivir deprisa, sin posibilidad de poner el tiempo de nuestro lado, sin poder aposentarnos en el tiempo. Hacer y entender la (teoría de la) educación de este nuestro mundo tecnocultural obliga también a recuperar los tiempos y los espacios como variables educativas, repensándolos. 
ÁNGEL GARCÍA DEL DUJO, JORIS VLIEGHE, JOSÉ MANUEL MUÑOZ Y JUDITH MARTÍN PENSAR LA (TEORÍA DE LA) EDUCACIÓN, DESDE LA TECNOLOGÍA DE NUESTRO TIEMPO

\section{CONSTRUCCIÓN DE CONOCIMIENTO EN ESPACIOS VIRTUALES - ... Y DE CONSTRUIR CONOCIMIENTO MIRANDO A TRAVÉS DE UNA PANTALLA}

Aquellos modos de reontologización de la realidad y de experienciación del mundo a través de las pantallas y estos procesos de construcción y reconstrucción de la identidad de los sujetos, cuando se ven y miran a sí mismos en esas pantallas, tienen que tener — nos gustaría pensar que casi necesariamente- su correlato en formas y procesos de conocimiento propios, característicos y probablemente distintos en algún sentido, de estos entornos virtuales; es ésta precisamente una de las líneas de investigación en la que tenemos depositadas más expectativas. Si, a lo largo de la historia, la introducción de artefactos y herramientas en la actividad humana trajo consigo cambios en las formas de generar conocimiento, no cabe esperar menos de esta tecnología digital, más bien al contrario, dadas las singularidades que presenta esta (r)evolución tecnológica a la que estamos asistiendo y que venimos señalando desde el principio. En resumen, una tecnología que está transformando la naturaleza intrínseca de nuestro mundo y reconfigurando nuestras formas de habitar y de actuar tanto en espacios físicos como en los virtuales (onlife) no puede por menos que afectar también los procesos de construcción del conocimiento.

En apartados anteriores hemos señalado, directa e indirectamente, algunos de los componentes de la acción educativa que se están viendo afectados por la re-ontologización a la que nos venimos refiriendo como catalizadora de los procesos varios de reconfiguración de los sujetos - las prácticas y formas de relación, los artefactos, los espacios y agentes intervinientes. Componentes todos ellos implicados en los procesos de construcción de conocimiento y que precisamente constituyen los puntales del andamiaje de la corriente socioconstructivista o vigotskiana (Ali, Joyes, \& Ellison, 2015; Daniels, 2003) en la que se apoyan aquellas teorías que han tratado de explicar cómo se genera el conocimiento de una forma acorde con la realidad y tecnología del momento. Un conjunto de planteamientos que coinciden en que el aprendizaje no puede entenderse fuera del contexto en el que tiene lugar $y$, en consecuencia, sus postulados se han centrado en ir superando las limitaciones contextuales, culturales, artefactuales, históricas y sociales propias de la época. No obstante, y a pesar de los intentos por adaptar el enfoque socioconstructivista a la realidad actual, los resultados que la investigación viene obteniendo no parecen lo suficientemente robustos para conformar un andamiaje teórico que respete y responda a las oportunidades que ofrece la tecnología de este tiempo (García del Dujo \& Martín-Lucas, 2020). ¿Cómo pensar ese andamiaje desde las reconfiguraciones y reontologizaciones que venimos advirtiendo? Ésta es la pregunta que ahora nos interesa.

Empezaremos por reconocer que quizás uno de los errores que venimos reproduciendo en el ámbito educativo es pensar sobre estas tecnologías desde una visión didáctica e instrumental, lo que dificulta la teorización acerca de los procesos de generación de conocimiento. Ésta es la razón por la que planteamos que el primer paso para avanzar en (teoría de la) educación es superar esta instrumentalidad, primero, porque no procede en estos momentos seguir reproduciendo 
y trasladando los escenarios tradicionales en los que se viene haciendo educación al entorno virtual, pues, como ya hemos señalado, esta tecnología está creando sus propios escenarios que vivimos y experienciamos como reales; cada vez cuesta más diferenciar entre actividades que realizamos online y offline (Floridi, 2014; 2015), razón por la que conviene buscar la convergencia entre el escenario tradicional (físico) y el nuevo (virtual). Y en segundo lugar, y en línea con la interpretación que Langdon Winner (1986) hace sobre la tecnología, conviene recordar que la tecnología que hemos introducido en los escenarios de aprendizaje no fue creada con fines educativos, por tanto, no debemos pensarla como un elemento neutral a nuestro servicio (Sánchez Rojo, 2017). En otras palabras, de la misma forma que un robot industrial no solo modifica la producción, sino que también redefine el significado del trabajo, la tecnología digital no solo modifica la forma de acceder o almacenar la información, sino que redefine la forma como generamos conocimiento, pues estamos hablando de tecnologías que interfieren e infiltran nuestros pensamientos, sentimientos, percepciones y patrones de conducta.

Por todo ello, y en la pretensión de comprender qué es lo que está cambiando en los procesos de aprendizaje en entornos virtuales, vamos a explorer aquí dos perspectivas que, a nuestro juicio, están determinando lo que podrían ser los cimientos de ese andamiaje teórico sobre las formas como se genera conocimiento en este nuevo ecosistema.

En primer lugar, en (teoría de la) educación deberíamos tener en cuenta una de las mayores características y potencialidades de esta tecnología, su autonomía. Para comprender mejor esta afirmación, tomaremos como referencia la interpretación que Luciano Floridi $(2010,2014)$ hace de estos artefactos tecnológicos, trasladándolo al ámbito educativo. Antes de que llegara la (r)evolución tecnológica que estamos presenciando, la tecnología podía clasificarse como artefacto primario o secundario en función de si mediaba entre el ser humano y la naturaleza o si lo hacía entre el ser humano y otro artefacto. Sirvan de ejemplos, respectivamente, un paraguas que media entre nosotros y la lluvia o unas tijeras que median entre un sastre y la tela. No sin razón afirma este autor, que los recientes y futuros avances tecnológicos de nuestras sociedades ofrecen artefactos que han conseguido dejarnos al margen de esa interacción ser humano — naturaleza - tecnología, sirva de ejemplo el smartphone que a través de la $a p p$ de nuestra entidad bancaria se comunica con un datafono que, a su vez, lo hace con la plataforma digital de un banco para confirmar la disposición de dinero. Este planteamiento, en términos pedagógicos, nos hace replantear el papel que la tecnología juega en los procesos de construcción de conocimiento. Nos referimos al hecho de que la autonomía que adquiere esta tecnología puede traducirse en nuevas posibilidades a la hora de construir conocimiento; en palabras de autores como Gabriel P. Swarts (2019), tecnologías como Siri, Cortana o Google Now podrían actuar como asistentes del educador en los procesos de aprendizaje y facilitar así una atención más personalizada.

Pues bien, si el rol que esta tecnología puede jugar en las acciones educativas está cambiando, cambiará también en consecuencia la explicación sobre las formas 
como se construye conocimiento en un mundo invadido por estos artefactos. Concretamente, si estamos ante una tecnología que cada vez adquiere mayor protagonismo en términos de autonomía, versatilidad y ubicuidad, quizás esta tecnología debiera ser comprendida más allá de su utilidad como actor técnico, como mero medio, situándose a la altura de actor interviniente, es decir, en un nivel superior a otras tecnologías predecesoras. Y conste que no nos estamos refiriendo al temor de que esta tecnología pueda sustituir al ser humano en el proceso de aprendizaje, pues, a nuestro parecer, un artefacto o herramienta tecnológica no puede tener agencia cognitiva en el sentido humano, en cuanto que no piensa, no razona, no opera, no funciona como lo hace el cerebro humano. No obstante, esto no impide atribuir un nivel de actuación superior a los artefactos terciarios frente a otras tecnologías anteriores, de manera que, en combinación sinérgica con la mente humana, puedan aflorar procesos distintos de conocimiento; precisamente, el hecho de considerar esta tecnología en la acción educativa más allá de un mero medio nos permite atisbar estas posibilidades, superando así la visión instrumental de la que hablábamos.

La segunda perspectiva que planteamos viene liderada por las implicaciones de esta tecnología en cuanto a su capacidad de extender nuestras capacidades cognitivas. Aunque la reflexión en el ámbito educativo en torno a si la tecnología extiende nuestras capacidades mentales no es nueva, pues los inicios de estos postulados se remontan a los pasados años 90 con la llegada de las teorías de la cognición distribuida (Hutchins, 1995) y de la mente extendida (Clark \& Chalmers, 1998), sí es cierto que en la última década se han producido avances importantes en los ámbitos teórico y empírico en base a estos planteamientos y fruto de la convergencia entre ramas de conocimiento como la pedagogía, la filosofía, la psicología y la neurociencia.

Pues bien, de un lado, ya Luciano Floridi (2010), en su interpretación sobre la vida informacional, advierte que estas tecnologías digitales e informacionales permiten liberarnos del esfuerzo mental que conllevan tareas de tipo superficial como resumir, memorizar, organizar o almacenar información, lo cual, trasladado al ámbito pedagógico, tiene serias implicaciones en los procesos de construcción de conocimiento. Y, si asumimos que estos artefactos no sustituyen, pero sí aumentan o extienden nuestras capacidades cognitivas, el primer paso sería tratar de explorar hasta qué punto los artefactos tecnológicos de nuestra época se acoplan a nuestros procesos mentales a la hora de generar conocimiento, pues no toda la tecnología lo hará al mismo nivel. Expresado en otras palabras, algunos artefactos pueden adquirir cierto status cognitivo si consiguen integrarse de manera profunda con nuestra cognición, no siendo así para aquellos otros artefactos que lo hicieran de manera superficial (Heersmink, 2015; 2017), v. gr., utilizar la señalización de los senderos para poder caminar por una ruta desconocida podría influir y ayudar a nuestra cognición para alcanzar nuestro objetivo, llegar a la cima de una montaña; sin embargo, personalizar la ruta a través de Google Maps o nuestra aplicación de GPS favorita sí podría considerarse co-constitutivo del proceso cognitivo de organizar y llevar a cabo esa ruta, porque requeriría de una interacción más compleja, 
profunda y duradera en el tiempo que la de servirnos de la señalización del camino. En definitiva, esta visión ofrece de nuevo una interpretación de la tecnología de nuestro tiempo en la que, si bien estos artefactos no pueden sustituir al sujeto, sí pueden adquirir cierto status cognitivo que les permita situarse en otro plano o nivel superior respecto del resto de tecnologías.

Por otra parte, esta consideración de la tecnología como extensión y liberación de algunas de nuestras capacidades cognitivas ha llevado a diferentes autores a estudiar y reflexionar sobre si su uso fomenta o socava nuestra forma de pensar de manera profunda. Autores como Nicholas Carr (2011) y estudios de carácter empírico como el realizado por Sparrow, Liu y Wegner (2011) argumentaban que el uso de la tecnología no nos permite pensar de manera profunda, sino que más bien nos invita a la superficialidad, pues nos facilitan no tener que recordar la información ni tampoco sintetizarla, ya que nos viene todo dado a través de las pantallas; en cambio, publicaciones más recientes, como las de Michael DeSchryver (2014; 2017), concretamente su Teoría de la Síntesis de Conocimiento mediado por la Web, argumentan que la tecnología digital fomenta el uso de habilidades de pensamiento de orden superior al ahorrarnos esfuerzo y tiempo en la realización de tareas de carácter más superficial como la búsqueda, memorización y almacenamiento de información. Estudios y reflexiones — a favor y en contra - que terminan coincidiendo en algunas consideraciones; esta tecnología proporciona nuevos soportes de aprendizaje que, al liberarnos de tareas de carácter más superficial — como memorizar, almacenar, procesar y distribuir información-, nos permiten centrarnos en tareas que requieren un mayor esfuerzo cognitivo - como consumir, sintetizar y crear; sin embargo, esta nueva realidad presenta también retos importantes y es que, si queremos aprovechar las potencialidades que estas tecnologías nos ofrecen, debemos adaptar nuestras metodologías y formas de entender la construcción de conocimiento, empezando por comprender qué de esta tecnología es lo que fomenta o impide nuestra manera de pensar de manera profunda y/o superficial a la hora de construir conocimiento (Heersmink \& Knight, 2018; Loh \& Kanai, 2016).

En todo caso, lo que venimos comentando evidencia que esta nueva forma de construir conocimiento propiciada por la tecnología de nuestro tiempo demanda formas diferentes de teorizar sobre unos fenómenos nuevos. Una tarea que, a nuestro parecer, debiera comenzar por superar la visión instrumental de estos artefactos, es decir, dejar de considerar estas tecnologías como meros actores técnicos para pasar a comprenderlas como actores intervinientes.

\section{CONCLUYENDO Y ABRIENDO PERSPECTIVAS A LA INVESTIGACIÓN}

Concluimos —en la línea de lo que venimos argumentando- que en estos momentos nuestro escenario de vida está dominado por una tecnología que está cambiando nuestra condición humana, nuestras formas de ser y de vivir, nuestras practicas y relaciones. En realidad, siempre lo estuvo por la tecnología del momento, en cuanto que es inherente a la condición humana su relación con el mundo mediada 
por una determinada tecnología, la que corresponde a cada época, pero quizás en la nuestra esos cambios o transformaciones estén siendo no solo más rápidos, más grandes y más amplios, sino distintos, de manera que la afectación humana puede terminar siendo diferente, no solo en magnitud y extensión, no solo de grado, sino de naturaleza diferente. Y ello referido a todos los órdenes de la realidad, es decir, una afectación integral del ser humano. También en lo que se refiere a educación.

Con este marco de referencia, concluimos que esta tecnología nuestra —uno de cuyos rasgos fundamentales viene dado por uno de sus componentes materiales más propios y característicos, las pantallas, de manera que hay cosas que vemos y otras que no vemos, tan importantes las unas como las otras, en ambos casos debido a procedimientos y mecanismos que no vemos, pero que no por ello deben quedar al margen de nuestra consideración, y las que vemos lo hacemos "modo pantalla", todo ello con fuertes implicaciones no solo fenomenológicas sino también ontológicas, epistemológicas y metodológicas - cambia nuestra forma de experienciar el mundo y, por ende, los modos de vernos y concebirnos a nosotros mismos y a los demás en permanente relación unos con otros y con lo otro, con las cosas, incluida nuestra forma de ver, de conocer, de sentir y comprender la realidad. Expresado en otros términos, lo que cambian son nuestras prácticas y formas de vida y con ello nuestros modos de ver, de hacer y de pensar la realidad, así como los modos como nos hacemos y pensamos unos con otros. Y todo ello mirando a través de una pantalla particular, con importantes implicaciones conceptuales y procedimentales.

Pues bien, en coherencia con este planteamiento, cabe concluir que también el mundo de la educación debe quedar (y de hecho queda) afectado por esta tecnología, incluyendo ahí los escenarios, los tiempos, los sujetos y, sobre todo, las prácticas, actividades y relaciones que dan sentido y conforman los procesos educativos y los modos como esta tecnología los cataliza y orienta. Como puede apreciarse, estamos yendo bastante más allá de una consideración meramente instrumental de esta tecnología, con todas sus derivadas, y, por ende, más allá también de una reflexión reduccionista de medios, fines y contenidos, por entender que ambas pertenecen a una época ya pasada y que esta tecnología tiene otros muchos nichos de reflexión más apropiados — e imprescindibles, diríamos nosotros- para construir una (teoría de la) educación de nuestro tiempo, por cuanto apuntan a una resignificación del concepto y de muchos de los términos y formas de pensamiento y acción en educación.

En esta intención hemos presentado aquí tres tópicos, relativos a los modos de ser, de conocer y de experienciar el mundo a través de la pantalla, con potencialidad suficiente para reorientar la investigación en (teoría de la) educación. No son los únicos, por supuesto; hemos elegido estos tres porque se corresponden con sendas líneas de investigación de los autores y porque, de alguna manera, sobre ellos descansa y pivota una teoría de la educación de este nuestro tiempo digital. Detras de estos tres epígrafes se esconden cuestiones de notable y amplia trascendencia, aunque inicialmente parece que solo sean de tipo epistemológico y metodológico, 
en las que nos encontramos trabajando y que, en sintonía con otros muchos autores (Vansieleghem, Vlieghe \& Zahn, 2019), enunciamos en forma de preguntas:

- Un primer bloque de cuestiones, por otra parte estrechamente entrelazadas, afloran al tomar los aspectos epistemológicos como punto de partida: si lo que se ve, se siente y se conoce a través de las pantallas, reontologiza la realidad que constituye el habitat del sujeto, ¿qué fue del concepto moderno de verdad, desplazado ahora quizás por el de utilidad?, ¿y de sus correlatos de unidad, coherencia, historia y progreso?, ¿se barrunta ya alguna situación post de las actuales identidades digitales múltiples, diversas y hasta de conveniencia, siempre efímeras y pasajeras?, ¿cómo compensa el sujeto, en sentido individual y colectivo, el resquebrajamiento que viene conociendo como individuo y ciudadano?, ¿cómo es el habitat de una ontología de lo actual y del momento, del tú y del yo ahora, más bien que del nosotros?, ¿significa esto en educación la destradicionalización del sujeto, como individuo y ciudadano, en conocimientos, lenguajes y formas de pensamiento?, ¿qué parte corresponde en esa operación a los lenguajes y modos digitales (metadatos) de percepción y construcción del sujeto, de la realidad y del conocimiento de sí mismo y de esa realidad?, ¿es suficiente en (teoría de la) educación con reorientar las actividades, los procesos y los currículos hacia el conocimiento de las gramáticas de lo digital, de su anatomía y fisiología, para someterlo a crítica filosófica, política y económica, no sin antes haberlo normativizado pedagógicamente, como suele ser habitual en educación, o se require más bien, dadas las importantes transformaciones que conlleva una sociedad poco a poco post-digital, una consideración de la educación en su condición política, en el sentido estricto y primario de convivencia y organización colectiva y democrática de la polis en base a la construcción de subjetividades, individuales y colectivas, siempre sociales?, ¿hay alguna otra salida humana y humanista a la altura de esta nuestra sociedad en constante progresion digital?, ¿cómo reconstruir en estos entornos las dimensiones del tiempo y del espacio, una y otra en toda su extensión, imprescindibles ambas para la constitución, configuración y desarrollo del sujeto y que siempre desempeñaron un importante papel en el carácter situado del proceso educativo (García del Dujo, 2009)?, ¿cómo abordar en estos escenarios la materialidad de las cosas, de los artefactos y objetos culturales, de los paisajes,... - aspectos estos recuperados en estos momentos para la investigación por otras disciplinas (Criado-Boado, Clark, Martínez Otero \& Müller, 2020), para asombro de la pedagogía-, de manera que concedamos a las cosas el sitio que les corresponde en la construcción del sujeto y, por ende, en su educación, y que la reflexion teórica casi siempre les negó, ignorándolas? Y una última pregunta en la que confluyen muchas de las anteriores: ¿¿cómo recomponer el circuito cuerpo-mente en los entornos virtuales, que siempre fue el soporte del proceso educativo y cuya ruptura por el propio concepto, naturaleza y mecanismos de lo digital mirando la 
pantalla deja al sujeto a la intemperie del pantanoso territorio virtual, como si de repente se hubiera quedado huérfano de algo?

- En otro orden de cosas, ahora de tipo metodológico, pero con impacto colateral en los ámbitos epistemológico y ontológico, procede preguntarnos si la potencialidad cuantitativa (big data) y cualitativa (creativa, en procesos y resultados de pensamiento y afectos) de esta tecnología, en los nuevos y particulares escenarios que genera, puede abrir camino, también en educación, a nuevas metodologías en investigación. Nos estamos refiriendo, de un lado, a la necesidad de tomar en consideración en educación el fenómeno de los Big Data en su doble sentido, funcional y estructural (Daniel, 2019), en cuanto que puede verse como un nuevo paradigma de investigación más allá de las racionalidades tradicionales — positivista, hermenéutica, estética e histórico-crítica-, nuevas formas de empirismo (Kitchin, 2014) que están desafiando, junto con técnicas y desarrollos disciplinarios recientes, la reflexion en educación, sobre todo en campos como la construcción del conocimiento mediada por la tecnología de nuestro tiempo, donde, por otra parte, se atisban nuevas formas, en procesos y resultados, de pensamiento con trascendencia en las políticas, las pedagogías y las prácticas educativas (Buckingham Shum \& Luckin, 2019).

Preguntas todas abiertas a la investigación, de orden diverso, epistemológico, ontológico y metodológico, pero también ético y politico, estrechamente entrelazadas y con potencialidad suficiente para reorientar la investigación en (teoría de la) educación, inluido el propio concepto y sentido de educar y ser educado.

\section{REFERENCIAS BIBLIOGRÁFICAS}

Ali, M. F., Joyes, G., \& Ellison, L. (2015). Proposing the third generation of an aligned activity system as a theoretical framework in blended learning research. Jurnal Teknologi, 75(3), 61-66. https://doi.org/10.11113/jt.v75.5044

Böhme, J. (2006). Schule am Ende der Buchkultur. Medientheoretische Begründungen schulischer Bildungsarchitekturen. Klinkhardt.

Buckingham Shum, S. J., \& Luckin, R. (2019). Learning analytics and AI: Politics, pedagogy and practices. British Journal of Educational Technology, 50(6), 2785-2793. https://doi. org/10.1111/bjet.12880

Cabero, J. (2016). ¿Qué debemos aprender de las pasadas investigaciones en Tecnología Educativa? Revista Interuniversitaria de Investigación en Tecnología Educativa, (0), 23-33. https://doi.org/10.6018/riite/2016/256741

Carbone, M. (2013). La vie mouvementée des écrans. Écrans, 1(3), 21-33.

Carr, N. (2011). Superficiales ¿Qué está haciendo internet con nuestras mentes? Taurus.

Clark, A., \& Chalmers, D. (1998). The Extended Mind. Analysis, 58(1), 7-19.

Cooley, H. (2004). It's all about the Fit: The Hand, the Mobile Screenic Device and Tactile Vision. Journal of Visual Culture, 3(2), 133-155. 
ÁNGEL GARCÍA DEL DUJO, JORIS VLIEGHE, JOSÉ MANUEL MUÑOZ Y JUDITH MARTÍN PENSAR LA (TEORÍA DE LA) EDUCACIÓN, DESDE LA TECNOLOGÍA DE NUESTRO TIEMPO

Costa, E. (2018). Affordances-in-practice: An ethnographic critique of social media logic and context collapse. New Media E Society, 20(10), 3641-3656. https://doi. org/10.1177/1461444818756290

Côté, J. E., \& Levine, C. G. (2002). Identity, Formation, Agency and Culture. A Social Psychological Synthesis. Lawrence Erlbaum Associates Publishers.

Criado-Boado, F., Clark, A., Martínez Otero, L. M., \& Müller, J. (2020). Material Minds: Exploring the Interactions between Predictive Brains, Cultural Artifacts and Embodied Visual Search. ERC Synergy Grants.

Dalmasso, A. C. (2020). Cadre et templum. Une archéologie des limites de l'image. La Part de l'Eil, 33, 133-151.

Daniel, B. (2019). Big Data and data science: A critical review of issues for educational research. British Journal of Educational Technology, 50(1), 99-111. https://doi.org/10.1111/ bjet.12595

Daniels, H. (2003). Vygotsky y la pedagogía. Paidós.

DeSchryver, M. (2014). Higher Order Thinking in an Online World: Toward a Theory of WebMediated Knowledge Synthesis. Teachers College Record, 116(12), 1-44.

DeSchryver, M. (2017). Using the Web as a Higher Order Thinking Partner. Journal of Educational Computing Research, 55(2), 240-271. https://doi.org/10.1177/0735633116667356

Dunbar, R. (2010). How many friends does one person need? Faber\& Faber.

Floridi, L. (2010). Information: a very short introduction. Oxford University Press.

Floridi, L. (2014). The 4th revolution: How the Infosphere is Reshaping Human Reality. Oxford University Press.

Floridi, L. (2015). The Onlife Manifesto: Being Human in a Hyperconnected Era (L. Floridi, ed.). https://doi.org/10.1007/978-3-319-04093-6

Fogel, J. F., \& Patino, B. (2013). La condition numérique. Grasset \& Fasquelle.

Flusser, V. (2011). Does writing have a future? (Nancy Ann Roth, Trans). University of Minnesota Press.

García del Dujo, A. (2009). Análisis del espacio en los entornos virtuales de formación. Teoría de la Educación. Revista Interuniversitaria, 21(1), 103-128. https://doi.org/10.14201/3157

García del Dujo, Á., y Martín-García, A. V. (2002). Caracterización pedagógica de los entornos virtuales de aprendizaje. Teoría de la Educación. Revista Interuniversitaria, 14, 67-92. https://doi.org/10.14201/2976

García del Dujo, Á., \& Martín-García, A. V. (2005). Pedagogical Analysis of a Virtual Learning Environment: The Tutorpea Project. En F. J. García, J. García, M. López, R. López \& E. Verdú (Eds.), Educational Virtual Spaces in Practice. The Odiseame Approach (pp. 205-218). Ariel.

García del Dujo, Á. \& Martín-Lucas, J. (2020). Towards 'onlife' education. How Technology is Forcing Us to Rethink Pedagogy. En A. V. Martín-García (Ed.), Blended Learning; Convergence between Technology and Pedagogy (pp. 1-19). Springer.

Goffman, E. (2001). La presentación de la persona en la vida cotidiana. Amorrortu.

Greenfield, S. (2014). Mind Change: How digital technologies are leaving their mark on our brains. Random House.

Heersmink, R. (2015). Dimensions of integration in embedded and extended cognitive systems. Phenomenology and the Cognitive Sciences, 14(3), 577-598. https://doi.org/10.1007/ s11097-014-9355-1 
Heersmink, R. (2017). Distributed Cognition and Distributed Morality: Agency, Artifacts and Systems. Science and Engineering Ethics, 23(2), 431-448. https://doi.org/10.1007/ s11948-016-9802-1

Heersmink, R., \& Knight, S. (2018). Distributed learning: Educating and assessing extended cognitive systems. Philosophical Psychology, 31(6), 969-990. https://doi.org/10.1080/0 9515089.2018.1469122

Humphreys, L., Gill, P., Krishnamurthy, B., \& Karnowski, V. (2013). Evolving Mobile Media: Uses and Conceptualisations of the Mobile Internet. Journal of Computer-Mediated Communication, 18(4), 491-507.

Hutchins, E. (1995). Cognition in the wild. MIT Press.

Introna, L., \& Ilharco, F. (2006). On the Meaning of Screens: Towards a Phenomenological Account of Screenness. Human Studies, 29(1), 57-76.

Kitchin, R. (2014). Big Data, new epistemologies, and paradigm shifts. Big Data \& Society, April-June, 1-12. https://doi.org/10.1177/2053951714528481

Loh, K. K., \& Kanai, R. (2016). How Has the Internet Reshaped Human Cognition? The Neuroscientist, 22(5), 506-520. https://doi.org/10.1177/1073858415595005

Memon, A., Sharma, S., Mohite, S., \& Jain, S. (2018). The role of online social networking on deliberate self-harm and suicidality in adolescents: A systematized review of the literature. Indian Journal of Psychiatry, 60(4), 384-392.

Pangrazio, L. (2019). Technologically situated: the tacit rules of platform participation. Journal of Youth Studies, 22(10), 1308-1326.

Sánchez Chamorro, D. X., Zamora Becerra, J., \& Cuellar Arenas, J. (2020). Las dos caras del nuevo prosumidor 2.0 ¿héroe o asesino de marcas? Colección Académica de Ciencias Sociales, 3(1), 83-94.

Sánchez Rojo, A. (2017). El derecho humano a la privacidad desde el enfoque de las capacidades: una reflexión educativa. EDETANIA, Estudios y Propuestas Socioeducativas, (51), 157-170.

Schofield, D., \& Kupiainen, R. (2015). Young People's Narratives of Media and Identity: Mediagraphy as Identity Work in Upper Secondary School. Nordicom Review, 36(1), 79-93.

Serres, M. (2012). Petite Poucette. Editions le Pommier.

Sparrow, B., Liu, J., \& Wegner, D. M. (2011). Google Effects on Memory: Cognitive Consequences of Having Information at Our Fingertips. Science, 333(6043), 776-778. https:// doi.org/10.1126/science.1207745

Stiegler, B. (2008). Prendre soin de la jeunesse et des générations. Flammarion.

Swarts, G. P. (2019). The School of "Onlife»: How Technology and Educational Data Will Force Us to Rethink Teaching and Learning. Teachers College Record. https://www.tcrecord. org/content.asp?contentid $=22648$

Tisseron, S. (2010). Cultures du livre et des écrans. La cohabitation indispensable. Le Carnet PSY 144.

Vlieghe, J. (2019). Education and world disclosure in the age of the screem. In N. Vansieleghem, J. Vlieghe \& Zahn, M. (Eds.), Education in the Age of Screen (pp. 23-35). Routledge.

Winner, L. (1986). The Whale and the Reactor. A search for Limits in an Age of High Techno$\log y$. Chicago Press. 